\title{
Characterization of Hybrid Communication Channel in Indoor Scenario
}

\author{
Thiago R. Oliveira, Fernando J. A. Andrade, Antonio A. M. Picorone, Haniph A. Latchman, Sergio L. Netto, and \\ Moises V. Ribeiro
}

\begin{abstract}
This work focuses on the characterization of indoor hybrid power line communication (PLC)-wireless channels in the frequency band between 1.7 and 100 MHz. These hybrid channels allow the simultaneous exploitation of the ubiquitous PLC channel and the mobility benefits offered by the wireless signals radiating from and being induced into power cables. A comprehensive study and analysis was conducted based on: (i) coherence time, (ii) additive noise power spectral density, (iii) coherence bandwidth, (iv) delay spread, (v) average channel gain, (vi) channel frequency response and (vii) channel capacity. Based on the reported analysis, the magnitude responses of hybrid PLC-wireless channels can be assumed to be symmetrical and significantly frequency selective. Also, we reveal that additive noise power spectral density and, consequently, channel capacity differ considerably in the PLC-to-wireless and wireless-to-PLC transmission directions. Finally, we show that the measured PLCwireless channels present a channel capacity of up to hundreds mega bits per second.
\end{abstract}

Index Terms-Channel characterization, power line communication, wireless communication, channel capacity, coherence time.

\section{INTRODUCTION}

$\mathbf{T}$ HE use of electric power grids to provide data communication (power line communication - PLC) has received significant attention. As these types of systems are entirely based on the existing and ubiquitous electric power infrastructure, significantly reductions to associated implementation costs are achieved in comparison to traditional telecommunications technologies [1]. Following this trend, a great deal of attention is currently being paid to the characterization of broadband (indoor, such as residences and buildings) [2] and narrowband (indoor or outdoor, such as for smart grid applications) [3], [4] data-communication channels. Additionally, there are efforts to characterize the electric power grids in vehicles (ships, spacecraft, airplanes, cars) [5]-[9] as well as in hostile environments, such as mine facilities and offshore

The Ad Hoc Associate Editor coordinating the review of this manuscript and approving it for publication was Prof. Renato Machado.

T. R. Oliveira (thiago.oliveira@ifsudestemg.edu.br) is with the Electronics Department, Federal Institute of Education, Science and Technology of the Southeast of Minas Gerais (IFSEMG), Campus Juiz de Fora, Brazil.

F. J. A. Andrade (fjaandrade@gmail.com), and A. A. M. Picorone (picorone@ieee.org) are with the Electrical Engineering Department, Federal University of Juiz de Fora (UFJF), Brazil.

M. V. Ribeiro (mribeiro@ieee.org) is with the Electrical Engineering Department, Federal University of Juiz de Fora (UFJF), and Smarti9 Ltda, Brazil.

H. A. Latchman (latchman@list.ufl.edu) is with the Department of Electrical \& Computer Engineering, University of Florida, US.

S. L. Netto (sergioln@smt.ufrj.br) is with the Electrical Engineering Program at COPPE-Federal University of Rio de Janeiro (UFRJ), Brazil.

Digital Object Identifier: 10.14209/jcis.2016.20 oil platforms [10], [11]. These investigations are resulting in a comprehensive understanding of such communication medium leveraging the introduction of PLC standards and technologies.

In a PLC system a physical connection is required between the PLC modem and the power cables by using, for instance, the power outlets. Therefore, mobility, which is the main attraction in wireless communications, is impractical in standard PLC systems and constitutes a major disadvantage of them. On the other hand, unshielded cabling infrastructure of electric power grids can radiate and be irradiated by wireless signals. Based on this intrinsic feature of power line cables, a new communication medium can be defined as hybrid PLC-wireless channel, consisting of unshielded power cables belonging to the electric power grids and the air. This observation leads to a new paradigm that can potentially renew the R\&D efforts in PLC technology by exploiting the interaction between the standard PLC and wireless technologies to provide mobility what can result in the so-called hybrid PLC-wireless technology.

Regarding PLC and wireless systems, we point out that the exploitation of the diversity offered by both of them has been recently addressed. Basically, the signal transmission occurs through the PLC and wireless channels in order to increase the system coverage or reliability by adopting some cooperative scheme. For instance, [12] shows results from the evaluation of some diversity combining schemes when a simultaneous data communication over wireless and PLC links is performed, with both data communication systems operating at their regulated frequency bands. Other similar investigations can be found in [13]-[15] and references therein.

Different from the aforementioned works, this paper addresses a communication medium established between a PLC device that makes use of the wireline (power cables) to receive/transmit signals with a wireless device, which use the air for data communication. The characterization of such kind of data communication medium was initially addressed in [16], [17]. Also, [18] discussed the usefulness of such communication medium incorporating a commercial PLC modem. A comprehensive characterization of the hybrid PLC-wireless channel for bi-directional data communication is timely and of utmost importance to precisely quantify the possibilities and potentials of such novel and challenging data-communication medium.

Therefore, this work presents a statistical characterization of the hybrid PLC-wireless channel, based on a measurement campaign, carried out on several medium-size apartments and residences, in the frequency band from $1.7 \mathrm{MHz}$ up to $100 \mathrm{MHz}$. Statistical analyses of average channel gain 
(ACG), coherence bandwidth (CB), coherence time (CT), root mean squared delay spread (RMS-DS), and channel capacity highlight the limitations, restriction, and potential of this data communication medium. Furthermore, the symmetry of the hybrid PLC-wireless channel magnitude response is verified when the transmitter and the receiver have the same access impedance (i.e., $50 \Omega$ ). In addition, differences between the noise power spectral densities from wireline (PLC) and from the wireless devices are reported. To the author's best knowledge, this is the first attempt to provide a complete and comprehensive characterization of hybrid PLC-wireless channels, for useful bidirectional communication.

The remainder of this work is organized as follows: the hybrid PLC-wireless channel and problem formulation are addressed in Section II. Section III briefly describes the measurement setup and campaign. In Section IV the parameters of interest for characterization of the hybrid PLC-wireless channels are briefly presented. Results and analyses are discussed in Section V. Finally, concluding remarks and suggestions for further research are presented in Section VI.

\section{The Hybrid PlC-Wireless Channel: Problem FORMULATION}

The main idea behind the hybrid PLC-wireless channel lies in the fact that the infrastructure of electric power grids consists mainly by unshielded cables. As a result, the power cables radiate signals and, conversely, wireless signals are inductively injected into them. These signal interactions have been treated in the realms of unwanted interference from and into electric power grids, see [19]-[22] and references therein. However, these usually unwanted interferences can be seen as useful signals that may potentially carry information that can be exchanged among wireline and wireless devices, leading to the hybrid PLC-wireless scenario illustrated in Fig. 1. In this framework, a PLC signal that is coupled into and propagated along the power cables is also radiated, and can be sensed by a nearby wireless device, which is connected to an antenna. On the other direction, a signal radiated in the air by the wirelessdevice antenna can, in part, be induced in unshielded power cables and reach a PLC device. Thus, a useful full-duplex communication channel can be established between the PLC and wireless devices through the hybrid PLC-wireless channel, as depicted Fig. 1, where both wireline and wireless devices are operating in the same frequency band.

In fact, the hybrid PLC-wireless channel introduces mobility to the PLC systems that is the main disadvantage of them if compared with wireless-based ones. We point out that the following application motivates the investigation of this novel communication medium:

- in vehicles, such as cars, ships and aircrafts, that make use of PLC systems, the hybrid PLC-wireless system can facilitate the interaction/maintenance of the devices, since the access to the power cables can be difficult and/or expensive;

- in home networks (HN) the hybrid PLC-wireless system seems to be more convenient from the consumer point of view than purely PLC systems, since in the last one a physical connection to the power cable is required;
- safety monitoring/control/maintenance of smart grids, mainly those that involve high voltage levels. In fact, the connection in a purely PLC system can be dangerous and expensive. By using the hybrid principle, the electric utility can easily access information and interact with the electric power grids.

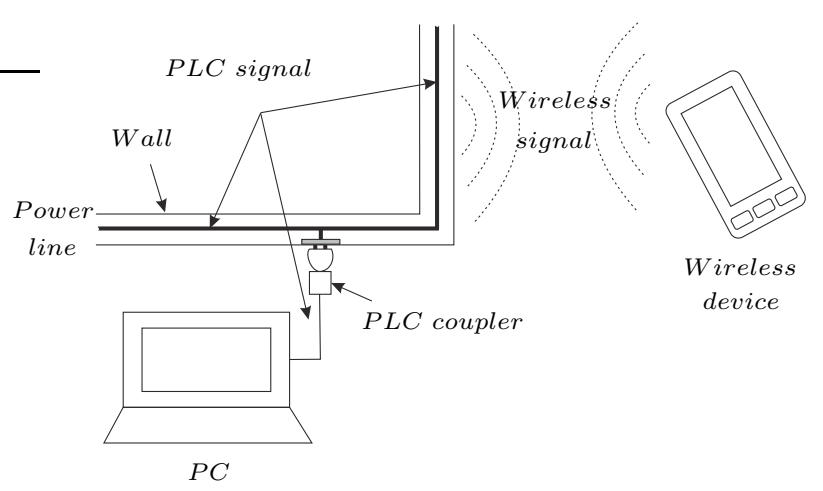

Fig. 1: Proposed hybrid PLC-wireless scenario.

Basically, a hybrid PLC-wireless communication system is composed of the following components (see Fig. 2):

- hybrid-PLC transceiver, which is connected to the outlet and responsible for injecting/receiving signals into/from the electric power grid;

- PLC coupler, which is a high-pass analog filter that promotes the interface between the PLC transceiver and the electric power grid, blocking the main frequency to prevent damage to the hybrid-PLC transceiver;

- hybrid PLC-wireless channel, which constitutes the communication medium established between the hybrid-PLC and hybrid-wireless transceivers;

- hybrid-wireless transceiver, namely the device that makes use of the wireless channel to provide data communication;

- antenna, which is the transducer of the hybrid-wireless transceiver that is designed to inject/receive signals into/from the wireless channel.

By assuming that the hybrid PLC-wireless channel is linear and time varying, its output can be written as

$$
y_{p w}(t)=\int_{0}^{t} x(\tau) h_{p w}(t, \tau) d \tau+w(t),
$$

for the signal propagation from the PLC-to-wireless device and,

$$
y_{w p}(t)=\int_{0}^{t} x(\tau) h_{w p}(t, \tau) d \tau+v(t),
$$

for the reverse path from the wireless-to-PLC device. Note that $h_{p w}(t, \tau)$ and $h_{w p}(t, \tau)$ denote the two channel responses at time $t$ when an impulse at instant $\tau$ is applied to the PLCto-wireless and wireless-to-PLC directions, respectively; $x(\tau)$ is an input signal; $w(t)$ and $v(t)$ are, respectively, the additive noise components in the PLC-to-wireless and wireless-to-PLC transmission directions.

By considering this new hybrid PLC-wireless channel for data communication purposes, it is crucial the knowledge of 
their features that affects the transmission of communications signals. In this way, this work addresses the characterization of the hybrid PLC-wireless channel through the analysis of measured data set obtained with the measurement setup described in Section III.

\section{Measurement Setup And CAMPAign}

The block diagram of the adopted measurement setup is depicted in Fig. 2 [23]. From this measurement setup, both coupler and antenna are considered as part of the channel to be characterized, since they are part of a hybrid PLCwireless communication system. Nevertheless, the coupler was designed to offer very low and flat attenuation in the frequency band of interest (see Fig. 15a in [24]). Thus, in the face of the severe diversities presented by PLC channels (for instance, high attenuations), the influence of this kind of coupler in the measured channels can be considered negligible. The adopted antenna was an omnidirectional and monopole one designed to operate in the frequency band ranging from 1 $\mathrm{MHz}$ up to $1 \mathrm{GHz}$. This antenna seems to be more appropriate than a directional one because the signal to/from the wires irradiate in all directions. The hybrid-PLC and hybridwireless transceivers are rugged computers equipped with a high-speed data acquisition board and a high-speed arbitrary signal generation board that operate as receiver and transmitter, respectively.

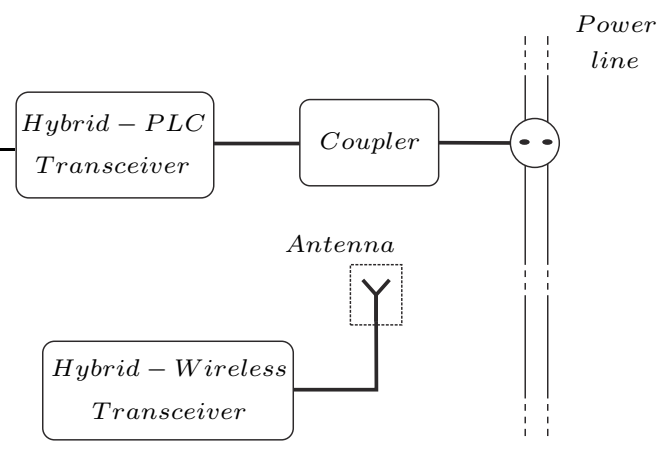

Fig. 2: Block diagram of the measurement setup.

The measurement setup makes use of a sounding technique, which is based on a orthogonal frequency division multiplexing (OFDM) scheme [25]. Basically, an off-line designed sounding signal is loaded into the memory of signal generation board, which injects the analog version of the sounding signal into the electric power grid (PLC to wireless direction) or the air (wireless to PLC direction). At the receiver end, the attenuated and distorted version of the transmitted sounding signal is acquired and recorded by the data acquisition board. Following a measurement methodology discussed in [24], the recorded signal is processed off-line using timing synchronization, sampling frequency error estimation and correction, channel estimation and channel estimation enhancement techniques to provide reliable estimates of the frequency response of hybrid PLC-wireless channels.

The parameters of the methodology that were adopted in the measurement campaign of the hybrid PLC-wireless channels are summarized in Tab. I, as detailed in [25]. In the applied methodology the channel is considered time invariant during the time interval corresponding to one transmitted symbol, which is supposed to be shorter than the coherence time of the hybrid PLC-wireless channel. By using the sounding estimation technique discussed in [24], each channel estimate is obtained every $23 \mu \mathrm{s}$. This time is much lower than the usual coherence time of indoor PLC channels, which is about $600 \mu \mathrm{s}$ [26]. Thus, $23 \mu \mathrm{s}$ seems to be a reasonable and conservative time interval for estimating hybrid PLC-wireless channels

TABLE I: Main Parameters Adopted by the Sounding Methodology and the Measurement Campaign.

\begin{tabular}{l|l}
\hline \hline Description & Value \\
\hline Sampling frequency & $f_{s}=200 \mathrm{MHz}$ \\
\hline Number of HS-OFDM symbols & 2 \\
\hline Number of sub-carriers & $N=2048$ \\
\hline Modulation & BPSK \\
\hline Cyclic prefix length & 512 \\
\hline Frequency resolution & $48.83 \mathrm{kHz}$ \\
\hline Symbol duration & $23.04 \mu \mathrm{s}$ \\
\hline
\end{tabular}

The locations in which the measurement campaign was carried out are listed in Tab. II. These facilities comprise typical residences and apartments in an urban area of Juiz de Fora, Brazil. Additional information about the measured places can be found in [27]. All potential scattering objects and the transceivers were stationary during the measurement campaign.

TABLE II: Main Features of the Chosen Measurement Places.

\begin{tabular}{c|c|c}
\hline \hline Construction type & Age (years) & Constructed area $\left(\mathrm{m}^{2}\right)$ \\
\hline House \#1 & 30 & 78 \\
\hline House \#2 & 10 & 69 \\
\hline Apartment \#1 & 9 & 54 \\
\hline Apartment \#2 & 9 & 42 \\
\hline Apartment \#3 & 18 & 65 \\
\hline Apartment \#4 & 3 & 62 \\
\hline Apartment \#5 & 2 & 54 \\
\hline
\end{tabular}

The following cases were considered during the measurement campaign:

- short-path channel: the wireless-PLC transceiver was randomly positioned within a $2-\mathrm{m}$ radius circle centered at the outlet in which the PLC-wireless transceiver is connected;

- long-path channel: the wireless-PLC transceiver was randomly placed into an area defined as a swept circle, having an outer and inner radius of $6 \mathrm{~m}$ and $2 \mathrm{~m}$, respectively, centered in the outlet in which the PLCwireless transceiver is connected.

By taking into account all facilities, 293 different combinations of locations for both PLC-wireless and wireless-PLC transceivers were evaluated. The wireless-PLC transceiver was positioned near to (short-path channel) and far from (longpath channel) the outlet in 200 and 93 combinations, respectively. Furthermore, approximately 600 estimates of the channel frequency response were measured for each combination. As a result, a total of 175,428 estimates of the hybrid PLCwireless channel frequency responses were obtained during 
the campaign. Additionally, additive noise in the localizations of both PLC-wireless and wireless-PLC transceivers were measured. In what follows we present the parameters extracted from the measured hybrid PLC-wireless channels and the obtained results.

\section{PARAMETERS DESCRIPTION}

The parameters used for characterizing the hybrid PLCwireless channels are described as follows.

\section{A. Average Channel Gain (ACG)}

The ACG is expressed by

$$
\bar{G}=\frac{1}{B} \int_{B}|H(f)| d f,
$$

where $H(f)$ is the channel frequency response at the frequency $f$ and $B$ is the frequency bandwidth. It is usually presented in $\mathrm{dB}$ as $\bar{G}_{d B}=10 \log _{10} \bar{G}$. The average channel attenuation (ACA), given by $\bar{A}_{d B}=-\bar{G}_{d B}$, is adopted in this contribution.

The ACA values can give an indication of the level of attenuation presented in a given communication channel, and as a consequence, an estimate of the channel capacity that can be expected on average.

\section{B. Coherence Bandwidth (CB)}

The $\mathrm{CB}$ reflects how selective the channel frequency response is. The CB of PLC channels are traditionally estimated by using [28]

$$
R\left(\Delta_{f}\right)=\int_{B} H(f) H^{*}\left(f+\Delta_{f}\right) d f,
$$

in which $B$ denotes the frequency band from $f_{\min }$ up to $f_{\max }$, while $\Delta_{f}$ refers to the frequency resolution. From Table I, our measurements had $\Delta_{f} \approx 48.83 \mathrm{kHz}, f_{\min }=1.7 \mathrm{MHz}$, and $f_{\max }=100 \mathrm{MHz}$.

In this contribution the correlation levels of $0.9,0.7$ and 0.5 were considered and are denoted by $B_{09}, B_{07}$ and $B_{05}$, respectively.

The CB is a key parameter used to evaluate the need for equalization and/or coding to deal with dispersive multipath effects.

\section{Root Mean Squared Delay Spread (RMS-DS)}

The RMS-DS represents the distribution of the transmitted power over various paths in a multipath environment, and can be defined as the square root of the second central moment of a power delay profile. The power delay profile of a channel impulse response (CIR) $h(t)$ can be calculated with

$$
P(t)=\frac{|h(t)|^{2}}{\int_{-\infty}^{\infty}|h(t)|^{2} d t} .
$$

The resulting RMS-DS is given by

$$
\sigma_{\tau}=\int\left(\sigma-\sigma_{e}-\sigma_{A}\right)^{2} P(\sigma) d \sigma
$$

where $\sigma_{A}$ corresponds to the time delay of the first transmitted signal at the receiver and $\sigma_{e}$ is the mean excess delay given by

$$
\sigma_{e}=\int\left(\sigma-\sigma_{A}\right) P(\sigma) d \sigma .
$$

Such channel feature indicates how dispersive the communication channel is. This information is usually used to support the specification of the guard interval duration in a multicarrier modulation to avoid intersymbol interference.

\section{Relation between $C B$ and RMS-DS}

The relation between the CB and the RMS-DS is generally expressed (in units of microseconds) by

$$
\sigma_{\tau} \approx \frac{\gamma}{B_{09}}
$$

in which $\gamma$ depends on the channel type and $B_{09}($ in $\mathrm{kHz})$ is the $\mathrm{CB}$ at a correlation level equal to 0.9 .

\section{E. Coherence Time $(C T)$}

The CT, denoted by $T_{c}^{\beta}$, is the time duration in which the CIR can be considered time invariant.This channel feature is crucial, for instance, to indicate the periodicity in which the channel state information must be estimated in order to perform effective equalization and resource allocation.

The correlation performed between consecutive channel impulses responses (CIR) is used in [29] to quantify the temporal variation of the channel. In this sense, at a certain value of the correlation level (threshold), the channel can be considered time invariant. Thus, as in [29], let

$$
T_{c}^{\beta}=M_{c}\left(2 N+L_{c p}\right) T_{s},
$$

where $M_{c}$ is the number of channel measurements needed to reach a correlation equal to $\beta, T_{s}$ denotes the sampling period, $N$ is the number of subcarrier in a HS-OFDM symbol, and $L_{c p}$ is the length of the cyclic prefix. In this contribution the CT at correlation levels of $\beta=\{0.85,0.90,0.95,0.99\}$ were adopted to analyze the variability of the hybrid PLC-wireless channel, although there is no indication in literature of the correlation level that is more suitable for our work.

\section{F. Channel Capacity}

The channel capacity of the Hybrid PLC-wireless channel was evaluated through [30]

$$
C=\max _{S_{x}(f)} \int_{B} \log _{2}\left(1+\frac{S_{x}(f)|H(f)|^{2}}{S_{N}(f)}\right) d f,
$$

where $B$ is the frequency bandwidth; $S_{x}(f)$ and $S_{N}(f)$ are the power spectral densities of the transmitted signal and the additive colored noise, respectively; and $\int_{B} S_{x}(f) d f=P_{x}$, where $P_{x}$ is the transmission power. The chosen equation for evaluating the channel capacity considers the hybrid PLCwireless channels to be frequency selective with the presence of the additive and colored noise. 


\section{RESUlts AND ANALYSES}

The presented results and analyses are based on the estimates of hybrid PLC-wireless channels obtained from the measured data in the frequency range from $1.7 \mathrm{MHz}$ up to $100 \mathrm{MHz}$. In addition, the frequency bands listed in Tab. III are considered to analyze the behavior of hybrid PLC-wireless channels in a regulated frequency band as well as in a frequency band that can be considered for future regulation efforts in favor of PLC and hybrid PLC-wireless technology.

TABLE III: Chosen sub-bands for analysis.

\begin{tabular}{c|c}
\hline \hline Notation & Frequency band (MHz) \\
\hline $\mathrm{FB}_{01}$ & $1.7-30$ \\
\hline $\mathrm{FB}_{02}$ & $30-70$ \\
\hline $\mathrm{FB}_{03}$ & $70-100$ \\
\hline $\mathrm{FB}_{T}$ & $1.7-100$ \\
\hline
\end{tabular}

\section{A. Channel Frequency Response Analysis}

1) Magnitude Response Symmetry: Measurements of channels were carried out in both PLC-wireless and wirelessPLC directions in order to verify the symmetry of hybrid PLC-wireless channels. Fig. 3 shows magnitude responses of four distinct but typical hybrid channel estimates - each one obtained from different combinations of two different positions of PLC-wireless and wireless-PLC transceivers. Fig. 3a shows results for wireless-PLC direction and Fig. $3 b$ for the reverse path. From these figures, the hybrid PLC-wireless channel can be seen to be symmetrical in terms of the magnitude frequency response, i.e., the magnitude response is independent of the transmission direction. This behavior is confirmed in all measured channels and agree with [31]. From the authors point of view, the minimal differences in Fig $3 c$ are due to uncertainty inherent of any measurement process. In spite of that, these minimal differences can be ignored since the curves for both transmission directions are very close. This symmetry property disagrees with what was reported in [16], most probably due to the PLC-coupler employed there which presented a poor frequency response (i.e. the attenuation considerably increases at higher frequencies). In the current work, however, a correctly designed PLC coupler was adopted, presenting an almost flat attenuation for the entire frequency range of interest (see Fig. 15a in [24]).

2) Influence of Small Distance Variations on the Magnitude Response: Fig. 4 shows four different scenarios where only the wireless-PLC transceiver was displaced in four different locations 0.4 meters, on average, apart. From this plot, one concludes that small position variations of the wireless-PLC transceiver can significantly affect the hybrid channel magnitude response for frequencies above $30 \mathrm{MHz}$.

3) Statistics: The maximum, minimum, mean, $50^{\text {th }}$ and $90^{t h}$ percentiles statistical parameters of the magnitude response of the measured hybrid PLC-wireless channels are depicted in Fig. 5. In Fig. 5a, which refers to short-path channels, the magnitude responses range from -5 to $-120 \mathrm{~dB}$, approximately, and $90 \%$ of observations stay within the magnitude range of -20 and $-40 \mathrm{~dB}$ for the frequency band ranging

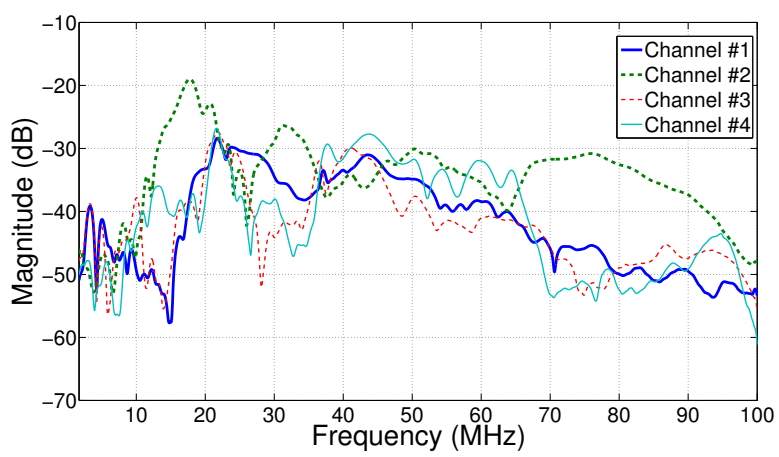

(a) Transmission from wireless-PLC to PLC-wireless transceiver.

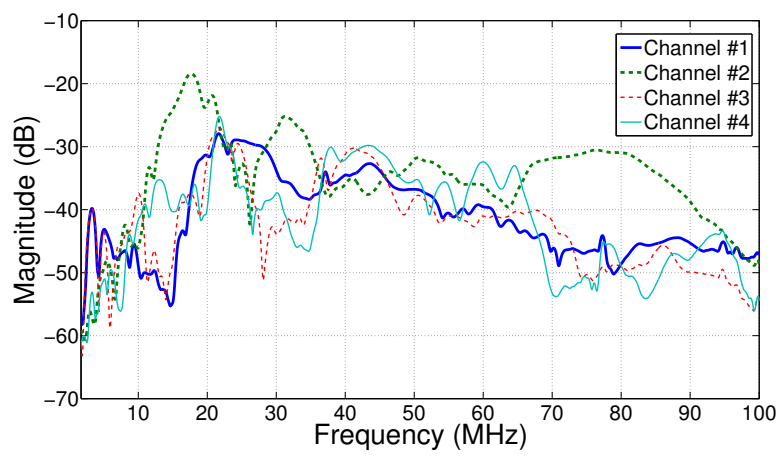

(b) Transmission from PLC-wireless to wireless-PLC transceiver.

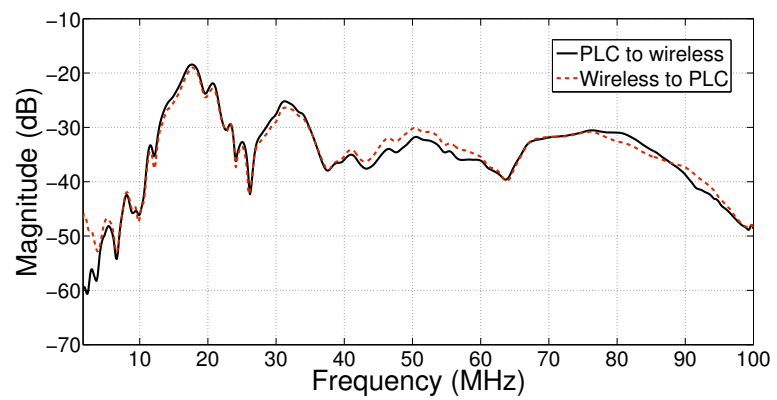

(c) A single channel in both directions.

Fig. 3: Magnitude responses of measured hybrid PLC-wireless channels in both transmission directions.

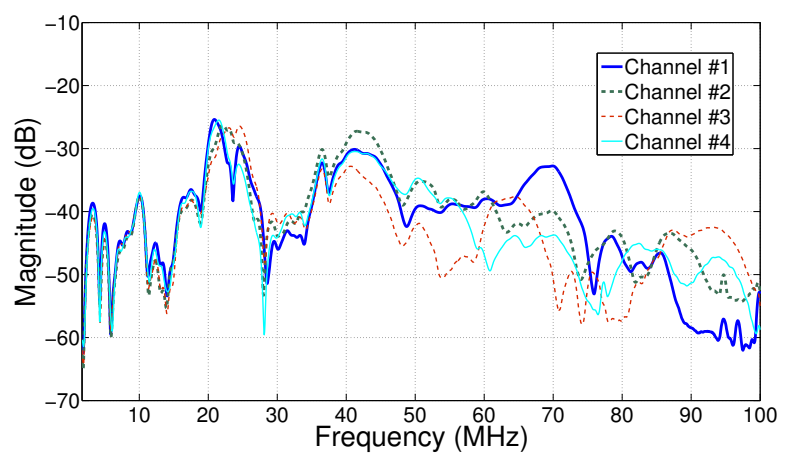

Fig. 4: Magnitude responses of some measured hybrid PLCwireless for different locations of the wireless-PLC transceiver. 
from 10 to $100 \mathrm{MHz}$. For long-path channels, as depicted in Fig. $5 \mathrm{~b}, 90 \%$ of the cases exhibit values below $-30 \mathrm{~dB}$, approximately, and in the frequency band from $1.7 \mathrm{MHz}$ up to $10 \mathrm{MHz}$, their values are below $-50 \mathrm{~dB}$. Comparing these two scenarios, one observes significant attenuation levels along long-path channels, as expected.

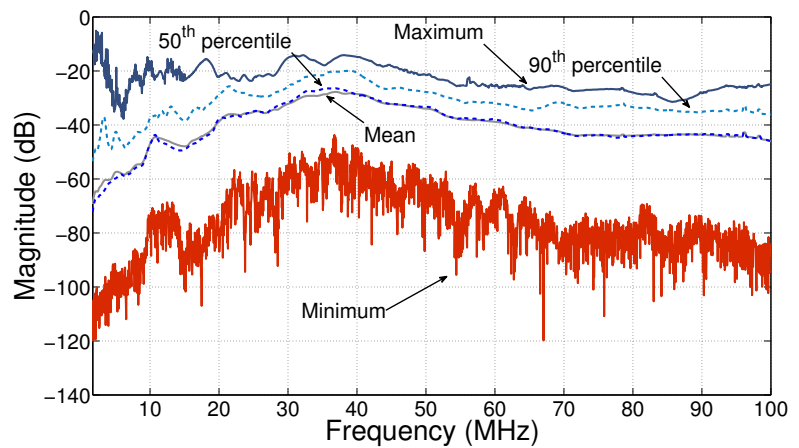

(a) Short-path channel.

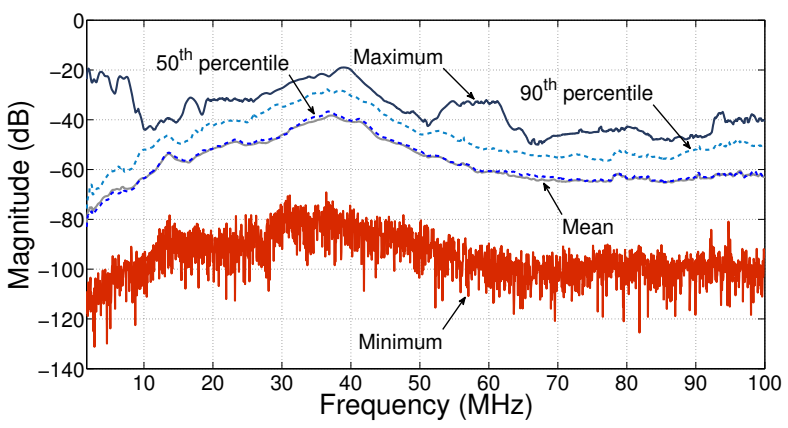

(b) Long-path channel.

Fig. 5: Statistical parameters extracted from estimated magnitude responses of the hybrid PLC-wireless channels.

\section{B. Average Channel Attenuation (ACA)}

ACA statistics are presented in Tab. IV, for frequency bands listed in Tab. III. Subband $\mathrm{FB}_{02}$ shows the lowest ACA achieving mean attenuation levels of 30 and $42 \mathrm{~dB}$ for the short- and long-path channels, respectively. On the other hand, subband $\mathrm{FB}_{03}$ presented the highest ACA values, reaching a maximum of $55.55 \mathrm{~dB}$ for short-path channels and $71.53 \mathrm{~dB}$ for long-path channels. Regarding the entire frequency band $\mathrm{FB}_{T}$ and considering short-path channel, ACA values are below $36.79 \mathrm{~dB}$ in $90 \%$ of the observations, while for longpath channel they are below $52.35 \mathrm{~dB}$. In general, these results emphasize that magnitude responses of hybrid PLC-wireless channels show significantly high attenuation levels.

Considering some reported results for the PLC scenario, we can verify that the mean ACA for short-path channels is less than that reported for indoor PLC channels in US [2] $(41.5 \mathrm{~dB})$. On the other hand, the mean attenuation observed in long-path channels are higher than those reported for indoor PLC channels in US.

\section{Coherence Bandwidth (CB)}

Table V summarizes some statistics associated with the estimated coherence bandwidths for measured PLC-wireless channels, considering correlation levels of $0.5\left(B_{05}\right), 0.7\left(B_{07}\right)$ and $0.9\left(B_{09}\right)$. In general, subband $\mathrm{FB}_{03}$ shows the highest mean $\mathrm{CB}$ values when compared to subbands $\mathrm{FB}_{01}$ and $\mathrm{FB}_{02}$ for short-path channels. For long-path channels the highest mean value is observed in subband $\mathrm{FB}_{02}$. For instance, for a correlation level of 0.5 in short-path channels, the mean values for the $\mathrm{CB}$ were of $4.95 \mathrm{MHz}, 10.09 \mathrm{MHz}$ and $12.32 \mathrm{MHz}$ for $\mathrm{FB}_{01}, \mathrm{FB}_{02}$ and $\mathrm{FB}_{03}$, respectively, while the mean of $\mathrm{CB}$ for long-path channels were of $3.26 \mathrm{MHz}, 4.08 \mathrm{MHz}$ and $1.33 \mathrm{MHz}$ for $\mathrm{FB}_{01}, \mathrm{FB}_{02}$ and $\mathrm{FB}_{03}$.

The maximum observed CB, for short-path channels, for the correlation levels of $0.5,0.7$ and 0.9 were, respectively, $57.96 \mathrm{MHz}, 30.45 \mathrm{MHz}$ and $13.91 \mathrm{MHz}$. For long-path channels, the maximum values of CB were $29.93 \mathrm{MHz}, 11.77 \mathrm{MHz}$ and $3.27 \mathrm{MHz}$ for the correlation levels of $0.5,0.7$ and 0.9 , respectively.

For a correlation level of 0.9 in short-path channels, subband $\mathrm{FB}_{02}$ shows $\mathrm{CB}$ values greater than $1.51 \mathrm{MHz}$ for $50 \%$ of observations, while the minimum $\mathrm{CB}$ value is $1.27 \mathrm{MHz}$ and $0.68 \mathrm{MHz}$ for subbands $\mathrm{FB}_{03}$ and $\mathrm{FB}_{01}$, respectively, for the same percentage level. For long-path channels, $\mathrm{CB}$ values for subbands $\mathrm{FB}_{01}$ and $\mathrm{FB}_{03}$ are greater than $0.29 \mathrm{MHz}$ and $0.27 \mathrm{MHz}$, respectively, while for subband $\mathrm{FB}_{02}$ the $\mathrm{CB}$ is greater than $0.98 \mathrm{MHz}$ in $50 \%$ of observations.

Considering subband $\mathrm{FB}_{T}$ and a correlation level of 0.9 , the mean $\mathrm{CB}$ value of $1.62 \mathrm{MHz}$ with a standard deviation of $0.66 \mathrm{MHz}$ is observed for short-path channels. Also, a $\mathrm{CB}$ value greater than $1.42 \mathrm{MHz}$ is observed in $50 \%$ of the observations and greater than $2.57 \mathrm{MHz}$ in only $10 \%$ of the estimates. On the other hand, long-path channels presents a mean $\mathrm{CB}$ value of $1 \mathrm{MHz}$ with a standard deviation of $505.64 \mathrm{kHz}$ and a $\mathrm{CB}$ value greater than $0.93 \mathrm{MHz}$ and $1.61 \mathrm{MHz}$ for $50 \%$ and $10 \%$, respectively, of the observations.

Also, we observed that long-path channels are more selective than its short-path counterpart, as expected. This behavior is verified in all considered correlation levels. Also, the minimal value was $48.83 \mathrm{kHz}$, for several evaluated scenarios. However, since this value is the frequency resolution (see Tab. I), the CB value could, eventually, be lower than the frequency resolution.

Finally, we compared the $\mathrm{CB}\left(B_{09}\right)$ of long-path and shortpath channels with the results reported for PLC channels in Spain [32] $\left(\mathrm{FB}_{01}\right)$ and France [28] $\left(\mathrm{FB}_{T}\right)$. While $B_{09}$ is higher than $0.2 \mathrm{MHz}$ in $50 \%$ of the cases in Spain, this value is $0.68 \mathrm{MHz}$ and $0.29 \mathrm{MHz}$ for short-path and long-path channels, respectively. For France, the mean value observed for $B_{09}$ was of $300 \mathrm{kHz}$, approximately, while for short-path and long-path channels $B_{09}$ was of $1.62 \mathrm{MHz}$ and $1.00 \mathrm{MHz}$, respectively. For the sake of conciseness, in the remainder of this contribution, only the $\mathrm{CB}$ value at a correlation level of $0.9\left(B_{09}\right)$ will be considered.

\section{Root Mean Squared Delay Spread (RMS-DS)}

Statistical parameters of RMS-DS are presented in Tab. VI. In this case, the subband $\mathrm{FB}_{02}$ exhibited the lowest RMS- 
TABLE IV: ACA for the Measured Hybrid PLC-Wireless Channels.

\begin{tabular}{|c|c|c|c|c|c|c|c|}
\hline & \multicolumn{7}{|c|}{$\mathrm{ACA}(\mathrm{dB})$} \\
\hline & Frequency band & Maximum & Minimum & Mean & Standard deviation & $50 \%$ below & $90 \%$ below \\
\hline \multirow{4}{*}{$\begin{array}{c}\text { Short-path } \\
\text { channel }\end{array}$} & $\mathrm{FB}_{01}$ & 50.57 & 24.39 & 36.38 & 4.68 & 36.72 & 41.94 \\
\hline & $\mathrm{FB}_{02}$ & 42.12 & 20.94 & 30.77 & 3.79 & 30.98 & 36.02 \\
\hline & $\mathrm{FB}_{03}$ & 55.55 & 26.65 & 41.69 & 5.41 & 42.32 & 48.28 \\
\hline & $\mathrm{FB}_{T}$ & 41.69 & 24.61 & 33.03 & 3.17 & 33.04 & 36.79 \\
\hline \multirow{4}{*}{$\begin{array}{c}\text { Long-path } \\
\text { channel }\end{array}$} & $\mathrm{FB}_{01}$ & 63.54 & 21.83 & 48.99 & 7.79 & 49.92 & 57.94 \\
\hline & $\mathrm{FB}_{02}$ & 64.73 & 28.64 & 42.66 & 5.92 & 42.14 & 49.62 \\
\hline & $\mathrm{FB}_{03}$ & 71.53 & 28.49 & 56.14 & 5.61 & 55.79 & 63.33 \\
\hline & $\mathrm{FB}_{T}$ & 63.06 & 27.11 & 44.89 & 6.33 & 45.29 & 52.35 \\
\hline
\end{tabular}

TABLE V: CB for the Measured Hybrid PLC-Wireless Channels.

\begin{tabular}{|c|c|c|c|c|c|c|c|}
\hline & \multicolumn{6}{|c|}{$\mathrm{CB}(\mathrm{kHz})$} \\
\hline & & Maximum & Minimum & Mean & Standard deviation & $50 \%$ below & $90 \%$ below \\
\hline \multirow{12}{*}{$\begin{array}{c}\text { Short-path } \\
\text { channel }\end{array}$} & $B_{05}\left(\mathrm{FB}_{01}\right)$ & $16.45 \times 10^{3}$ & 97.66 & $4.95 \times 10^{3}$ & $3.62 \times 10^{3}$ & $4.05 \times 10^{3}$ & $10.47 \times 10^{3}$ \\
\hline & $B_{05}\left(\mathrm{FB}_{02}\right)$ & $39.99 \times 10^{3}$ & 244.14 & $10.09 \times 10^{3}$ & $7.72 \times 10^{3}$ & $7.23 \times 10^{3}$ & $18.75 \times 10^{3}$ \\
\hline & $B_{05}\left(\mathrm{FB}_{03}\right)$ & $29.93 \times 10^{3}$ & 97.66 & $12.32 \times 10^{3}$ & $9.43 \times 10^{3}$ & $8.74 \times 10^{3}$ & $29.32 \times 10^{3}$ \\
\hline & $B_{05}\left(\mathrm{FB}_{T}\right)$ & $57.96 \times 10^{3}$ & 97.66 & $8.67 \times 10^{3}$ & $6.78 \times 10^{3}$ & $6.73 \times 10^{3}$ & $14.65 \times 10^{3}$ \\
\hline & $B_{07}\left(\mathrm{FB}_{01}\right)$ & $10.84 \times 10^{3}$ & 97.66 & $2.49 \times 10^{3}$ & $1.82 \times 10^{3}$ & $1.90 \times 10^{3}$ & $5.08 \times 10^{3}$ \\
\hline & $B_{07}\left(\mathrm{FB}_{02}\right)$ & $30.45 \times 10^{3}$ & 195.31 & $4.58 \times 10^{3}$ & $3.79 \times 10^{3}$ & $3.71 \times 10^{3}$ & $7.47 \times 10^{3}$ \\
\hline & $B_{07}\left(\mathrm{FB}_{03}\right)$ & $29.93 \times 10^{3}$ & 48.83 & $6.55 \times 10^{3}$ & $6.82 \times 10^{3}$ & $4.44 \times 10^{3}$ & $13.57 \times 10^{3}$ \\
\hline & $B_{07}\left(\mathrm{FB}_{T}\right)$ & $27.49 \times 10^{3}$ & 48.83 & $4.40 \times 10^{3}$ & $2.95 \times 10^{3}$ & $3.76 \times 10^{3}$ & $6.59 \times 10^{3}$ \\
\hline & $B_{09}\left(\mathrm{FB}_{01}\right)$ & $3.76 \times 10^{3}$ & 97.66 & $0.81 \times 10^{3}$ & $0.54 \times 10^{3}$ & $0.68 \times 10^{3}$ & $1.32 \times 10^{3}$ \\
\hline & $B_{09}\left(\mathrm{FB}_{02}\right)$ & $6.05 \times 10^{3}$ & 97.66 & $1.57 \times 10^{3}$ & $6.02 \times 10^{3}$ & $1.51 \times 10^{3}$ & $2.29 \times 10^{3}$ \\
\hline & $B_{09}\left(\mathrm{FB}_{03}\right)$ & $13.91 \times 10^{3}$ & 48.83 & $1.75 \times 10^{3}$ & $1.90 \times 10^{3}$ & $1.27 \times 10^{3}$ & $4.35 \times 10^{3}$ \\
\hline & $B_{09}\left(\mathrm{FB}_{T}\right)$ & $4.69 \times 10^{3}$ & 195.31 & $1.62 \times 10^{3}$ & $0.66 \times 10^{3}$ & $1.42 \times 10^{3}$ & $2.57 \times 10^{3}$ \\
\hline \multirow{12}{*}{$\begin{array}{c}\text { Long-path } \\
\text { channel }\end{array}$} & $B_{05}\left(\mathrm{FB}_{01}\right)$ & $17.87 \times 10^{3}$ & 97.66 & $3.26 \times 10^{3}$ & $3.42 \times 10^{3}$ & $2.09 \times 10^{3}$ & $8.69 \times 10^{3}$ \\
\hline & $B_{05}\left(\mathrm{FB}_{02}\right)$ & $17.53 \times 10^{3}$ & 97.66 & $4.08 \times 10^{3}$ & $2.48 \times 10^{3}$ & $3.47 \times 10^{3}$ & $7.17 \times 10^{3}$ \\
\hline & $B_{05}\left(\mathrm{FB}_{03}\right)$ & $29.93 \times 10^{3}$ & 48.83 & $1.33 \times 10^{3}$ & $2.93 \times 10^{3}$ & $2.11 \times 10^{3}$ & $8.00 \times 10^{3}$ \\
\hline & $B_{05}\left(\mathrm{FB}_{T}\right)$ & $12.01 \times 10^{3}$ & 146.48 & $3.84 \times 10^{3}$ & $2.14 \times 10^{3}$ & $3.47 \times 10^{3}$ & $6.59 \times 10^{3}$ \\
\hline & $B_{07}\left(\mathrm{FB}_{01}\right)$ & $11.77 \times 10^{3}$ & 97.66 & $1.43 \times 10^{3}$ & $1.43 \times 10^{3}$ & $1.12 \times 10^{3}$ & $2.98 \times 10^{3}$ \\
\hline & $B_{07}\left(\mathrm{FB}_{02}\right)$ & $6.93 \times 10^{3}$ & 48.89 & $2.32 \times 10^{3}$ & $1.09 \times 10^{3}$ & $2.20 \times 10^{3}$ & $3.56 \times 10^{3}$ \\
\hline & $B_{07}\left(\mathrm{FB}_{03}\right)$ & $7.52 \times 10^{3}$ & 48.83 & $0.38 \times 10^{3}$ & $0.90 \times 10^{3}$ & $0.88 \times 10^{3}$ & $4.34 \times 10^{3}$ \\
\hline & $B_{07}\left(\mathrm{FB}_{T}\right)$ & $6.10 \times 10^{3}$ & 48.83 & $2.27 \times 10^{3}$ & $1.19 \times 10^{3}$ & $2.19 \times 10^{3}$ & $3.86 \times 10^{3}$ \\
\hline & $B_{09}\left(\mathrm{FB}_{01}\right)$ & $3.27 \times 10^{3}$ & 48.83 & $0.40 \times 10^{3}$ & $0.39 \times 10^{3}$ & $0.29 \times 10^{3}$ & $0.93 \times 10^{3}$ \\
\hline & $B_{09}\left(\mathrm{FB}_{02}\right)$ & $2.78 \times 10^{3}$ & 48.83 & $0.99 \times 10^{3}$ & $0.48 \times 10^{3}$ & $0.98 \times 10^{3}$ & $1.51 \times 10^{3}$ \\
\hline & $B_{09}\left(\mathrm{FB}_{03}\right)$ & $2.29 \times 10^{3}$ & 48.83 & $0.11 \times 10^{3}$ & $0.14 \times 10^{3}$ & 273.44 & $1.41 \times 10^{3}$ \\
\hline & $B_{09}\left(\mathrm{FB}_{T}\right)$ & $2.78 \times 10^{3}$ & 146.48 & $1.00 \times 10^{3}$ & 505.64 & $0.93 \times 10^{3}$ & $1.61 \times 10^{3}$ \\
\hline
\end{tabular}

DS values in all evaluated scenarios, whereas subband $\mathrm{FB}_{03}$ presented the highest ones. RMS-DS values below $0.168 \mu \mathrm{s}$, $0.085 \mu$ s and $0.533 \mu$ s were observed in subbands $\mathrm{FB}_{01}, \mathrm{FB}_{02}$ and $\mathrm{FB}_{03}$, respectively, in $90 \%$ of observations for short-path channels. For long-path channels, the corresponding threshold values were $0.419 \mu \mathrm{s}, 0.179 \mu \mathrm{s}$ and $1.425 \mu \mathrm{s}$.

For the complete frequency band $\mathrm{FB}_{T}$, long-path channels exhibited a maximum value of $1.476 \mu$ s for the RMS-DS, whereas in short-path channels such value was $1.196 \mu \mathrm{s}$. In $90 \%$ of the cases, the RMS-DS was above $0.133 \mu$ s for short-path channels, compared with $0.331 \mu \mathrm{s}$ for long-path channels.

The RMD-DS mean value observed for both the shortpath and long-path channels are lower than that reported for indoor PLC channels in the urban area in US [2] $(0.23 \mu \mathrm{s})$, considering $\mathrm{FB}_{01}$.

\section{E. $C B$ versus $R M S-D S$}

The relation between $\mathrm{CB}$ and RMS-DS parameters was given by (8) as $\sigma_{\tau} \approx \gamma / B_{09}$. The observed relation for the hybrid PLC-wireless channel is shown in Fig. 6, where the dotted line denotes the case $\gamma=111$, which represents the best fit for short-path channels according to the minimum mean squared error (MMSE) criterion. The analysis for long-path channels returned $\gamma=106$, which is close to that achieved for short-path channels. For comparison purposes, reference [28] reported $\gamma=55$ for a standard PLC channel, whereas [33] provided $\gamma \approx 150$ and [34] reported $\gamma<100$ for some wireless channels.

It is interesting to note that the results observed from this inverse relation for both hybrid and pure PLC channels reflects that there is a relation between the channel frequency selectivity and the delay spread.

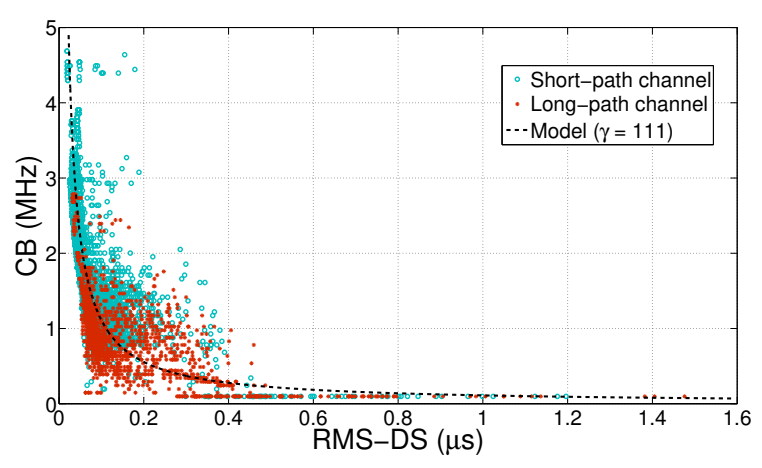

Fig. 6: Scatter plot of CB versus RMS-DS. 
TABLE VI: RMS-DS of the Measured Hybrid PLC-Wireless Channels.

\begin{tabular}{|c|c|c|c|c|c|c|c|}
\hline & \multicolumn{7}{|c|}{ RMS-DS $(\mu s)$} \\
\hline & Frequency band & Maximum & Minimum & Mean & Standard deviation & $50 \%$ below & $90 \%$ below \\
\hline \multirow{4}{*}{$\begin{array}{c}\text { Short-path } \\
\text { channel }\end{array}$} & $\mathrm{FB}_{01}$ & 1.685 & 0.024 & 0.129 & 0.064 & 0.122 & 0.168 \\
\hline & $\mathrm{FB}_{02}$ & 0.936 & 0.031 & 0.066 & 0.035 & 0.059 & 0.085 \\
\hline & $\mathrm{FB}_{03}$ & 1.857 & 0.013 & 0.180 & 0.288 & 0.056 & 0.533 \\
\hline & $\mathrm{FB}_{T}$ & 1.196 & 0.018 & 0.085 & 0.069 & 0.071 & 0.133 \\
\hline \multirow{4}{*}{$\begin{array}{l}\text { Long-path } \\
\text { channel }\end{array}$} & $\mathrm{FB}_{01}$ & 1.742 & 0.029 & 0.214 & 0.219 & 0.139 & 0.419 \\
\hline & $\mathrm{FB}_{02}$ & 1.540 & 0.025 & 0.108 & 0.094 & 0.079 & 0.178 \\
\hline & $\mathrm{FB}_{03}$ & 1.928 & 0.011 & 0.440 & 0.556 & 0.095 & 1.425 \\
\hline & $\mathrm{FB}_{T}$ & 1.476 & 0.019 & 0.162 & 0.147 & 0.107 & 0.331 \\
\hline
\end{tabular}

\section{F. Coherence Time (CT)}

The coherence time was estimated using the procedure described in [29]. The main parameters of the procedure are listed in Tab. VII. Basically, $K_{t}$ is the term used to truncate the measured CIR based on cumulative energy of their coefficients; $K_{s}$ is a factor in which the values of CIR below this threshold are replaced by zero, thus resulting in a sparse representation of the CIR; $K_{c}$ is a factor that discard the coefficients with less energy, which can distort the coherence time estimate. Furthermore, only those measurements that provided more than 640 consecutive estimates of frequency response of hybrid PLC-wireless channels were taken into account.

Figure 7 shows the correlation evolution for all measured hybrid PLC-wireless channels for short-path channels. Note that the $y$-axis refers to different channel configurations while the $x$-axis is the time evolution of the same communication medium through its several consecutive estimates and the correlation is evaluated with respect to the first channel impulse response as reference. We can see that the majority of channels have a similar temporal variability of its channel impulse response. The empirical cumulative distribution function (CDF) of the CT for short-path channels case is presented in Fig. 8, where different coherence levels are considered. This figure suggests that CT was below approximately $156 \mu$ s for $90 \%$ of the observed cases, considering the coherence level $(\beta)$ of 0.99 .

TABLE VII: Setup parameters for the coherence-time estimation procedure [29] based on the channel impulse response (CIR).

\begin{tabular}{l|c|c}
\hline \hline Description & Variable & Value \\
\hline Measured CIR energy \% (truncated CIR) & $K_{t}$ & 0.9 \\
\hline Coefficients amplitude \% (sparse CIR) & $K_{s}$ & $-40 \mathrm{~dB}$ \\
\hline Selecting the most relevant factors & $K_{c}$ & $-20 \mathrm{~dB}$ \\
\hline
\end{tabular}

Figure 9 shows the correlation evolution of the hybrid PLCwireless channel for long-path channels. In this case, the analyzed hybrid PLC-wireless channels have distinct and severe variations of temporal correlation. That behavior indicates that these channels present shorten time intervals than shortpath channels in which the channel impulse response can be considered time invariant, as confirmed by the coherence-time CDF plots depicted in Fig. 10. These plots also suggest that the CT for long-path channels is shorten than $39.5 \mu \mathrm{s}$, only one fourth of its short-path counterpart, in $90 \%$ of the observed cases, for $\beta=0.99$.

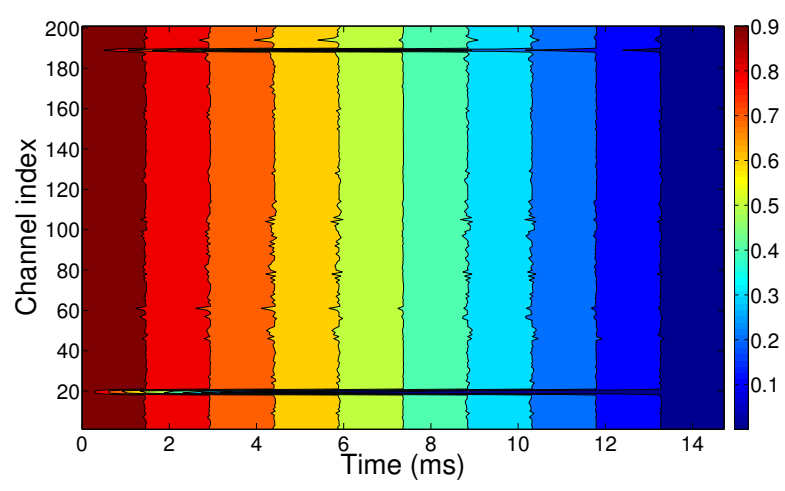

Fig. 7: Correlation evolution for the short-path channel.

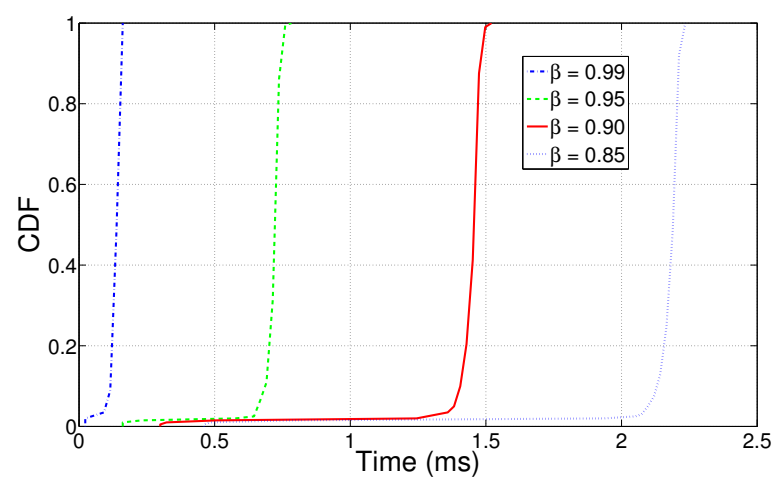

Fig. 8: CT CDF of the short-path channel for distinct correlation levels.

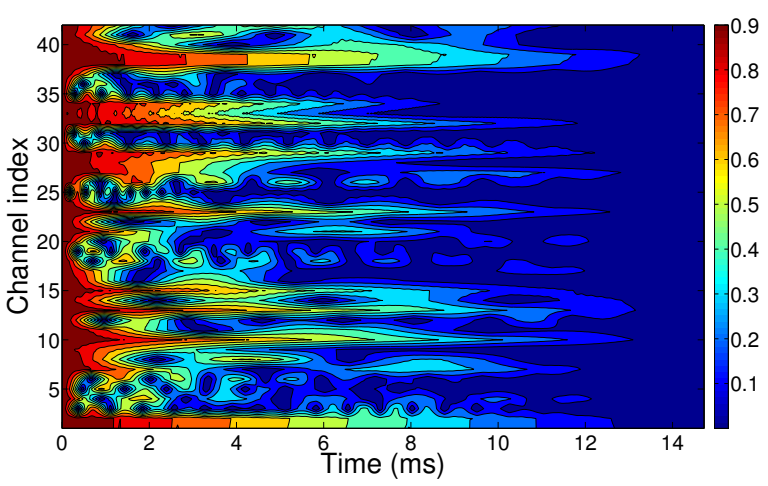

Fig. 9: Correlation evolution for long-path channel. 


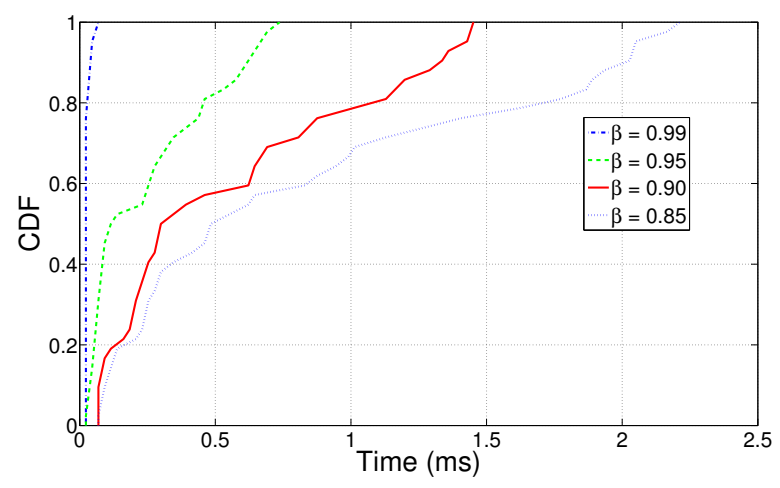

Fig. 10: CDF of CT for long-path channel by considering distinct values of correlation levels.

\section{G. Additive Noise}

Additive noise in both PLC and wireless interfaces was measured in order to estimate the channel capacity in the measured hybrid PLC-wireless channels. The statistical parameters of the measured noise are shown in Fig. 11 in terms of power spectral density (PSD), estimated by means of the Welch method described in [35]. Fig 11a reflects the noise behavior in the wireless channel, where the presence of narrowband noise, such as those from frequency modulation (FM) stations in frequencies around $100 \mathrm{MHz}$, is very clear. Considering the entire $\left(\mathrm{FB}_{T}\right)$ frequency band, background-noise PSD mean value was around $-114 \mathrm{dBm} / \mathrm{Hz}$.

Figure $11 \mathrm{~b}$ presents the noise measured in electric power grids, where the exponential behavior of background noise, which is typical in PLC channels, together with high energy in low frequencies is evident. Also, some high-frequency narrowband noise components can be identified, agreeing with those measured in the air. A comparison between PSDs obtained in both environments indicates that severe noise is observed in PLC channel, mainly for low frequencies, while less variations between minimum and maximum values are noticed for high frequencies.

\section{H. Channel Capacity}

The additive noise described in Subsection V-G was taken into account to estimate the channel capacity offered by the hybrid PLC-wireless channel, for the frequency band $\mathrm{FB}_{T}$. Fig. 12 shows the mean values of channel capacities for the short-path and long-path channels, respectively, considering both transmission directions (PLC-to-wireless and wireless-toPLC). For this plot, the channel capacity was evaluated with PSD transmission power ranging from -90 up to $-50 \mathrm{dBm} / \mathrm{Hz}$ in steps of $5 \mathrm{dBm} / \mathrm{Hz}$. As expected, short-path channels exhibits higher capacity than long-path channels. Also, higher capacities were observed in PLC-to-wireless transmission direction due to high power noise presence in the PLC channel. Thus, the hybrid PLC-wireless channels are asymmetrical from a channel capacity point of view, due to the additive noise.

Figure 13 shows the empirical complementary cumulative distribution function (CCDF) of the channel capacity for hybrid PLC-wireless in both transmission directions. In this case,

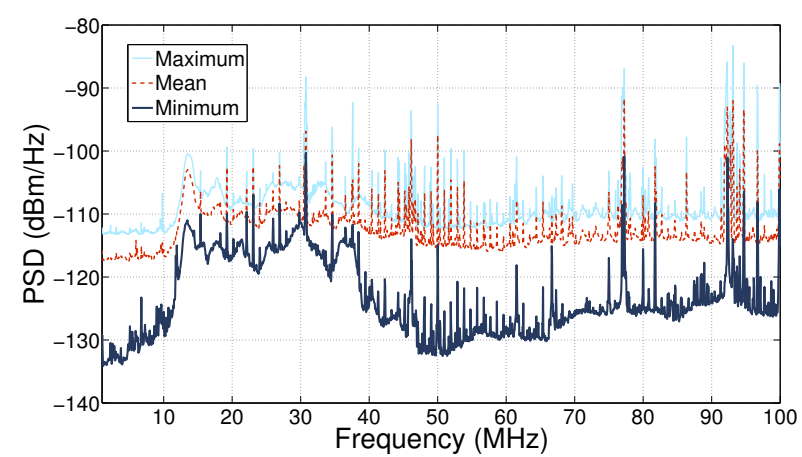

(a) PSD of the additive noise at the input of the hybrid-wireless transceiver.

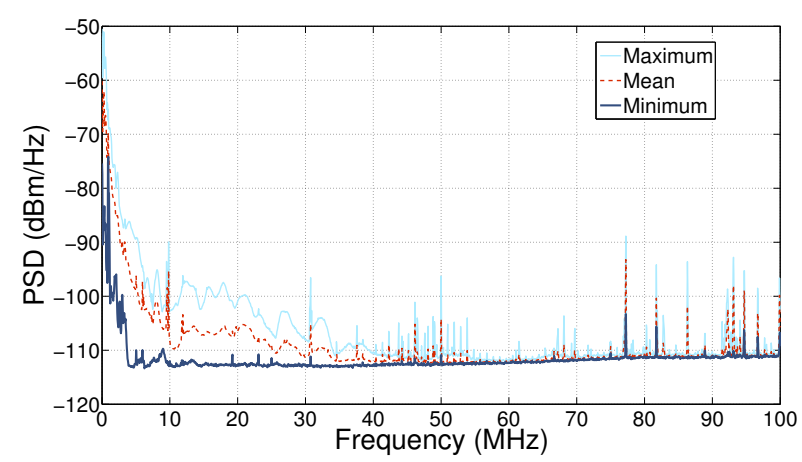

(b) PSD of the additive noise at the input of the hybrid-PLC transceiver.

Fig. 11: PSD of the additive noise in hybrid PLC-wireless channels.

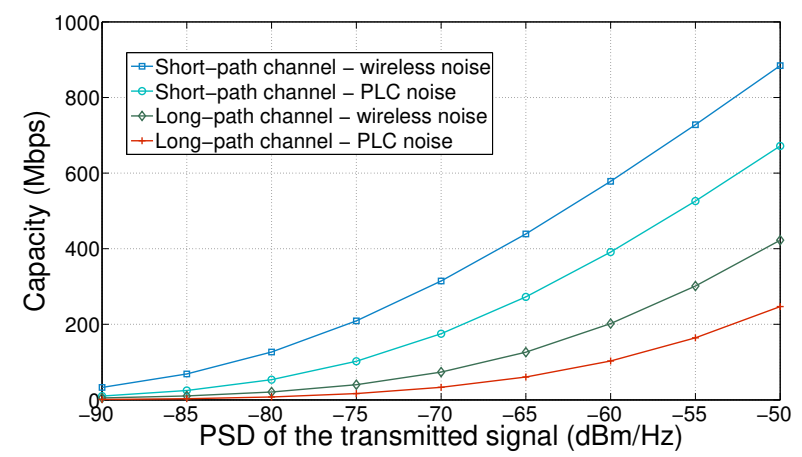

Fig. 12: Mean values of channel capacity for the short-path channel and long-path channel.

the PSD of the transmitted signal was made $-55 \mathrm{dBm} / \mathrm{Hz}$ in the frequency band from 1.7 up to $30 \mathrm{MHz}$ and $-80 \mathrm{dBm} / \mathrm{Hz}$ in the remainder frequencies. These adopted PSD levels for the transmitted signal agrees with the regulations for PLC systems [36]. For short-path channels, the minimal achieved capacity was $16 \mathrm{Mbps,}$ whereas for long-path channels the minimal capacity was around $3 \mathrm{Mbps}$. Also, it is interesting to note that channel capacity is higher than $150 \mathrm{Mbps}$ in $50 \%$ of the observed cases for the PLC-to-wireless direction of shortpath channels and can surpass $450 \mathrm{Mbps}$. If the transmission direction is considered, a difference of up to $139 \mathrm{Mbps}$ and $46 \mathrm{Mbps}$ in the capacity is observed for short-path channels and long-path channels, respectively. 


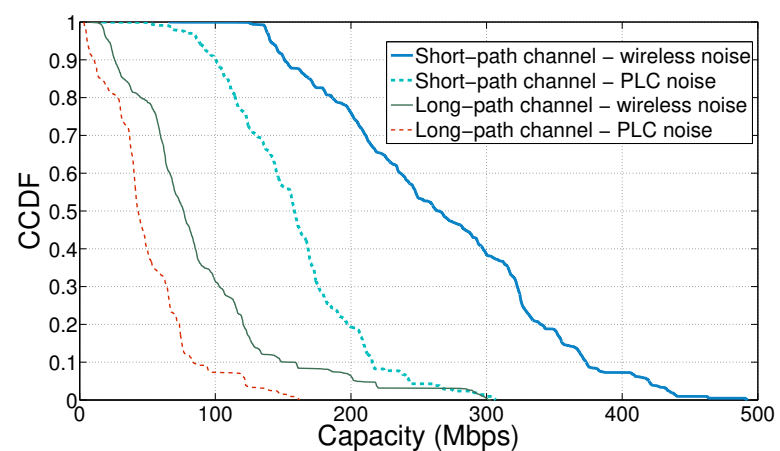

Fig. 13: CCDF of channel capacity for short-path channel and long-path channel.

\section{CONCLUSIONS}

This work focused on the characterization of the hybrid PLC-wireless channels that emerge from the exploitation of the benefits of the intrinsic and traditionally unwanted radiation in unshielded power cables to provide mobility in PLC systems.

Several statistical parameters of hybrid PLC-wireless channels were presented and analyzed, considering an entire frequency band ranging from $1.7 \mathrm{MHz}$ up to $100 \mathrm{MHz}$, as well as some subbands within it. Based on estimates of hybrid PLC-wireless channels obtained in a measurement campaign carried out inside two residences and five apartments, two scenarios (short-path and long-path channels) were devised and thoroughly analyzed. The statistical analyses showed that hybrid PLC-wireless channels are strongly frequency selective, exhibit high attenuation levels and present symmetrical magnitude responses (irrespective to the transmission direction).

Additionally, we verified that channel capacities surpassing $450 \mathrm{Mbps}$ can be achieved for short-path channel, when transmitter-received distances are below 2 meters. When this distance is increased, within a range from 2 to 6 meters, channel capacities above $85 \mathrm{Mbps}$ were still observed for more than $90 \%$ of measured hybrid PLC-wireless channels.

Finally, but not the least, we anticipate that this work can promote the development of PLC-based mobile and untethered communication applications such as in wireless sensor network, smart energy, intelligent buildings, and related areas.

\section{ACKNOWLEDGMENTS}

The authors would like to thanks IFSEMG, FINEP, FAPEMIG, CNPq, CAPES, P\&D ANEEL, CEMIG, INERGE, the Fulbright Commission and Smarti9 for their financial supports.

\section{REFERENCES}

[1] H. Hrasnica, A. Haidine, and R. Lehnert, Broadband PowerLine Communications Network Design. John Wiley \& Sons, 2005, doi: 10.1002/047002092X.ch1.

[2] S. Galli, "A novel approach to the statistical modeling of wireline channels," IEEE Trans. on Communications, vol. 59, no. 5, pp. 13321345, May 2011, doi: 10.1109/TCOMM.2011.031611.090692.

[3] S. Galli, A. Scaglione, and Z. Wang, "For the grid and through the grid: The role of power line communications in the smart grid," Proceedings of the IEEE, vol. 99, no. 6, pp. 998-1027, Jun. 2011, doi: 10.1109/JPROC.2011.2109670.
[4] W. Liu, M. Sigle, and K. Dostert, "Channel characterization and system verification for narrowband power line communication in smart grid applications," IEEE Communications Magazine, vol. 49, no. 12, pp. 2835, Dec. 2011, doi: 10.1109/MCOM.2011.6094003.

[5] T. Zheng, M. Raugi, and M. Tucci, "Time-invariant characteristics of naval power-line channels," IEEE Trans. on Power Delivery, vol. 27, no. 2, pp. 858-865, Apr. 2012, doi: 10.1109/TPWRD.2011.2181542.

[6] V. Degardin, I. Junqua, M. Lienard, P. Degauque, and S. Bertuol, "Theoretical approach to the feasibility of power-line communication in aircrafts," IEEE Trans. on Vehicular Technology, vol. 62, no. 3, pp. 1362-1366, Mar. 2013, doi: 10.1109/TVT.2012.2228245.

[7] V. Degardin, P. Laly, M. Lienard, and P. Degauque, "Investigation on power line communication in aircrafts," IET Communications, vol. 8, no. 10, pp. 1868-1874, Jul. 2014, doi: 10.1049/iet-com.2013.0828.

[8] M. Lienard, M. O. Carrion, V. Degardin, and P. Degauque, "Modeling and analysis of in-vehicle power line communication channels," IEEE Trans. on Vehicular Technology, vol. 57, no. 2, pp. 670-679, Mar. 2008, doi: 10.1109/TVT.2007.906372.

[9] I. S. Stievano, F. G. Canavero, W. R. Garcia Valverde, L. Guerrieri, and P. Bisaglia, "Multipath modeling of automotive power line communication channels," IEEE Trans. on Industrial Informatics, vol. 10, no. 2, pp. 1381-1391, May 2014, doi: 10.1109/TII.2014.2307199.

[10] J. Li, B. Whisner, and J. A. Waynert, "Measurements of mediumfrequency propagation characteristics of a transmission line in an underground coal mine," IEEE Trans. on Industry Applications, vol. 49, no. 5, pp. 1984-1991, Sep. 2013, doi: 10.1109/TIA.2013.2260812.

[11] J. Yazdani, K. Glanville, and P. Clarke, "Modelling, developing and implementing sub-sea power-line communications networks," in Proc. International Symposium on Power Line Communications and Its Applications, Apr. 2005, pp. 310-316, doi: 10.1109/ISPLC.2005.1430521.

[12] S. W. Lai and G. G. Messier, "Using the wireless and PLC channels for diversity," IEEE Trans. on Communications, vol. 60, no. 12, pp. 3865-3875, Dec. 2012, doi: 10.1109/TCOMM.2012.081512.110408.

[13] J.-H. Lee and Y.-H. Kinm, "Diversity relaying for parallel use of powerline andwireless communication networks," IEEE Trans. on Power Delivery, vol. 29, no. 3, pp. 1301-1310, Jun. 2014, doi: 10.1109/TPWRD.2013.2290034.

[14] M. Kuhn, S. Berger, I. Hammerstrom, and A. Wittneben, "Power line enhanced cooperative wireless communications," IEEE Journal on Selected Areas in Communications, vol. 24, no. 7, pp. 1401-1410, Jul. 2006, doi: 10.1109/JSAC.2006.874407.

[15] O. A. Gonzalez, J. Urminsky, M. Calvo, and L. De-Haro, "Performance analysis of hybrid broadband access technologies using PLC and WiFi," in Proc. International Conference on Wireless Networks, Communications and Mobile Computing, vol. 1, Jun. 2005, pp. 564-569, doi: 10.1109/WIRLES.2005.1549470.

[16] T. R. Oliveira, C. A. G. Marques, M. S. Pereira, S. L. Netto, and M. V. Ribeiro, "The characterization of hybrid PLC-wireless channels: A preliminary analysis," in Proc. IEEE International Symposium on Power Line Communications and Its Applications, Mar. 2013, pp. 98-102, doi: 10.1109/ISPLC.2013.6525832.

[17] T. R. Oliveira, F. J. A. Andrade, L. G. S. Costa, M. S. Pereira, and M. V. Ribeiro, "Measurement of hybrid PLC-wireless channels for indoor and broadband data communication," in Proc. XXXI Simpsio Brasileiro de Telecomunicaes, Sep. 2013, doi: 10.14209/sbrt.2013.184.

[18] A. S. de Beer, H. C. Ferreira, and A. J. Vinck, "Contactless powerline communications," in Proc. IEEE International Symposium on Power Line Communications and its Applications, Mar. 2014, pp. 111-115, doi: 10.1109/ISPLC.2014.6812366.

[19] M. Gebhardt, F. Weinmann, and K. Dostert, "Physical and regulatory constraints for communication over the power supply grid," IEEE Communications Magazine, vol. 41, no. 5, pp. 84-90, May 2003, doi: 10.1109/MCOM.2003.1200106.

[20] IEC 610003-8, "Electromagnect Compatibility (EMC) - Part 3: Limits - Section 8: Signaling on Low-Voltage Electrical Installations - Emission levels, Frequency bands and Electromagnect Disturbances levels", Geneva, Switzeland, 2002.

[21] FCC, "Code of Federal Regulations - Title 47: Telecommunication Chapter I: FCC Part 15 - Radio Frequency Devices", Washington, DC, 2002.

[22] CISPR 22, "Information Technology Equipment - Radio Disturbance Characteristics - Limits and Methods of Measurement", Geneva, Switzeland, 2002.

[23] G. R. Colen, C. A. G. Marques, T. R. Oliveira, F. P. V. de Campos, and M. V. Ribeiro, "Measurement setup for characterizing low-voltage and outdoor electric distribution grids for PLC systems," in Proc. IEEE PES 
Conference On Innovative Smart Grid Technologies Latin America, Apr. 2013, pp. 1-5, doi: 10.1109/ISGT-LA.2013.6554 476.

[24] T. R. Oliveira, C. A. G. Marques, W. A. Finamore, S. L. Netto, and M. V. Ribeiro, "A methodology for estimating frequency responses of electric power grids," Journal of Control, Automation and Electrical Systems, vol. 25 , no. 6, pp. 720-731, 2014, doi: 10.1007/s40313-014-0151-5.

[25] T. R. Oliveira, W. A. Finamore, and M. V. Ribeiro, "A sounding method based on OFDM modulation for PLC channel measurement," in Proc. IEEE International Symposium on Power Line Communications and Its Applications, Mar. 2013, pp. 185-190, doi: 10.1109/ISPLC.2013.6525 847.

[26] F. J. C. Corripio, J. A. C. Arrabal, L. D. del Rio, and J. T. E. Munoz, "Analysis of the cyclic short-term variation of indoor power line channels," IEEE Journal on Selected Areas in Communications, vol. 24, no. 7, pp. 1327-1338, Jul. 2006, doi: 10.1109/JSAC.2006.874402.

[27] Measured brazilian places. [Online]. Available: https://sites.google.com/a/ifsudestemg.edu.br/thiagooliveira/plc

[28] M. Tlich, A. Zeddam, F. Moulin, and F. Gauthier, "Indoor power-line communications channel characterization up to $100 \mathrm{MHz}$ - part II: Timefrequency analysis," IEEE Trans. on Power Delivery, vol. 23, no. 3, pp. 1402-1409, Jul. 2008, doi: 10.1109/TPWRD.2007.916095.

[29] A. A. M. Picorone, R. Sampaio-Neto, and M. V. Ribeiro, "Coherence time and sparsity of brazilian outdoor PLC channels: A preliminary analysis," in Proc. IEEE International Symposium on Power Line Communications and Its Applications, Mar. 2014, pp. 1-5, doi: 10.1109/ISPLC.2014.6812337.

[30] T. M. Cover and J. A. Thomas, Elements of Information Theory. John Wiley \& Sons, 2005, doi: $10.1002 / 047174882 \mathrm{X}$.

[31] S. Galli and T. Banwell, "A novel approach to the modeling of the indoor power line channel-part II: transfer function and its properties," IEEE Trans. on Power Delivery, vol. 20, no. 3, pp. 1869-1878, July 2005, doi: 10.1109/TPWRD.2005.848732.

[32] F. J. Canete, J. A. Cortes, L. Diez, and J. T. Entrambasaguas, "A channel model proposal for indoor power line communications," IEEE Communications Magazine, vol. 49, no. 12, pp. 166-174, Dec. 2011, doi: 10.1109/MCOM.2011.6094022.

[33] K. Pahlavan and S. J. Howard, "Frequency domain measurements of indoor radio channels," Electronics Letters, vol. 25, no. 24, pp. 16451647, Nov. 1989, doi: 10.1049/el:19891103.

[34] G. J. M. Janssen, P. A. Stigter, and R. Prasad, "Wideband indoor channel measurements and BER analysis of frequency selective multipath channels at 2.4, 4.75, and $11.5 \mathrm{GHz}$,' IEEE Trans. on Communications, vol. 44, no. 10, pp. 1272-1288, Oct. 1996, doi: 10.1109/26.539768.

[35] P. Welch, "The use of fast Fourier transform for the estimation of power spectra: A method based on time averaging over short, modified periodograms," IEEE Trans. on Audio and Electroacoustics, vol. 15, no. 2, pp. 70-73, Jun. 1967, doi: 10.1109/TAU.1967.1161901.

[36] B. Praho, M. Tlich, P. Pagani, A. Zeddam, and F. Nouvel, "Cognitive detection method of radio frequencies on power line networks," in Proc. IEEE International Symposium on Power Line Communications and Its Applications, Mar. 2010, pp. 225-230, doi: 10.1109/ISPLC.2010.5 479894.

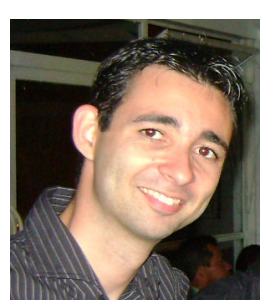

Thiago Rodrigues Oliveira received the B.S. (2007), M.Sc. (2010) and D.Sc. (2015) degrees from Federal University of Juiz de Fora (UFJF), all in Electrical Engineering. He has been a Professor at the Department of Electronics of the Federal Institute of Education, Science and Technology of the Southeast of Minas Gerais, Juiz de Fora, Brazil. His currently research works involve the following topics: digital signal processing, digital communication, internet of things, power line medium characterization.

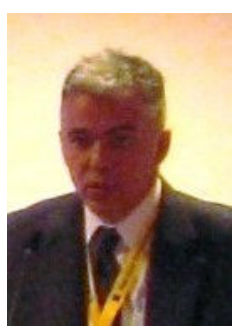

Fernando J. A. Andrade received the degrees in Electronics Engineering from the Federal University of Rio de Janeiro (UFRJ), Brazil, in 1989, Network Engineering and Communication Systems Specialist from National Institute of Telecommunications (Inatel), Brazil, in 2003, Master and Doctor in Electrical Engineering from Pontifical University of Rio de Janeiro (PUC-Rio), Brazil, in 2006 and 2010, respectively. He currently works as professor of Electrical Engineering in Federal University of Juiz de Fora (UFJF) and his main research interests are in radio propagation and wireless communications areas.

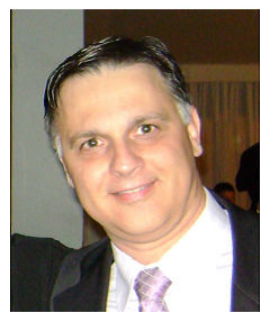

Antonio A. M. Picorone was born in São João Nepomuceno - MG, Brazil, in 1967. He has a Licentiate in Science (1999) and Post Graduation in Mathematics (2002) at Centro de Ensino Superior de Juiz de Fora (CES/JF), Juiz de Fora - MG (Brazil). He received B.Sc. (2007), M.Sc. (2009) and D.Sc. (2014) in Electrical Engineering from the Federal University of Juiz de Fora (UFJF), Brazil. Since 1989 he has been with Companhia Energética de Minas Gerais (CEMIG), where he is a supervisor in telecommunications. He has been a Professor at the Department of Electrical Engineering of the CES/JF since 2008. His main researches involve digital communications, compressive sensing, PLC channel modeling and estimation.

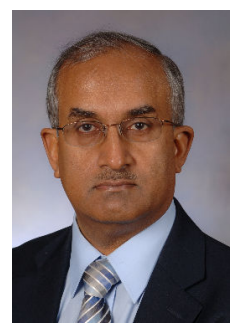

Dr. Haniph A. Latchman joined the University of Florida in 1987 where he is presently Professor of Electrical and Computer Engineering and teaches and conducts research in the areas of Control Systems, Communications and Computer Networks. Dr. Latchman received the D.Phil. from Oxford University in 1986 and the B.Sc. degree (First Class Honors) from the University of the West Indies (UWI)-Trinidad and Tobago, in 1981. He was selected as the 1983 Jamaica Rhodes Scholar as well as the 1978 recipient of the UWI Open Scholarship. Dr. Latchmans research and teaching focuses on multimedia, wireless and powerline communications and networks as well as on multivariable control system analysis and design. Dr. Latchman is also actively involved in the use of computer, communication and information technology to enhance the learning experience for traditional on-campus students as well as in distance education. Dr. Latchman is a Senior Member of the IEEE and has published some 180 technical journal articles and conference proceedings and four books in the general areas of Communication Networks and Control Systems. He has directed $24 \mathrm{Ph} . \mathrm{D}$. dissertations and 39MS theses and has served as Associate Editor and guest editor for several international journals, and as General Chair and member of technical program committees in the areas of communications and control systems and networks. Dr. Latchmans teaching and research has been recognized by numerous awards, including several Best Paper awards, the University of Florida Teacher of the Year Award, and the 2000 IEEE Undergraduate Teaching Award, the Boeing Summer Faculty Fellowship and 2001 and 2013 Fulbright Fellowships. Dr. Latchman was also selected as a Life Member of the University of Florida Academy of Distinguished Teaching Scholars in 2015 . 


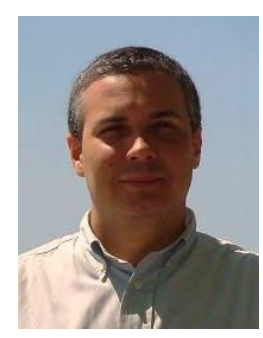

Sergio L. Netto was born in Rio de Janeiro, Brazil. He received the B.Sc. degree (cum laude) from the Federal University of Rio de Janeiro (UFRJ), Brazil, in 1991, the M.Sc. degree from COPPE/UFRJ in 1992, and the Ph.D. degree from the University of Victoria, BC, Canada, in 1996, all in Electrical Engineering. Since 1997, he has been an Associate Professor with the Department of Electronics and Computer Engineering, at Poli/UFRJ, and, since 1998, with the Program of Electrical Engineering, at COPPE/UFRJ. He is the co-author (with P. S. R. Diniz and E. A. B. da Silva) of "Digital Signal Processing: System Analysis and Design" by Cambridge University Press, 2nd ed., 2010. His teaching and research interests lie in the areas of digital signal processing, speech processing, information theory and math education.

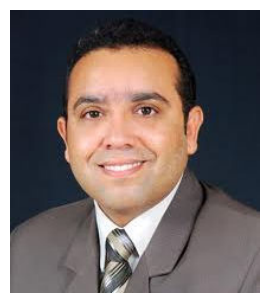

Moises V. Ribeiro received the B.S. degree in Electrical Engineering from the Federal University of Juiz de Fora (UFJF), MG, Brazil, and M.Sc. and D.Sc. degrees in Electrical Engineering from the University of Campinas, SP, Brazil, in 1999, 2001 and 2005, respectively. He was a Visiting Scholar at University of California in Santa Barbara, CA, USA, in 2004, Visiting Professor (2005-2007) and Assistant Professor (2007-2015) at UFJF. Since 2015, he has been an Associate Professor at UFJF. He co-founded Smarti9 Ltda and Wari Ltda in 2012 and 2015, respectively. His research interests includes signal processing, digital communication, power line communication, smart grids, internet of things and smart city. In these fields, he has authored over 150 peer reviewed papers, 9 book chapters, and filed 11 patents. He was the General Chair of the 2010 IEEE ISPLC, 2013 IWSGC, SBrT 2015, and a Guest Co-Editor for Special Issues in the EURASIP Journal on Advances in Signal Processing and EURASIP Journal of Electrical and Computer Engineering. He had served as the Secretary of the IEEE ComSoc TC-PLC. He was the recipient of Fulbright Visiting Professorship at Stanford University, Stanford, CA, USA, in 2011, and at Princeton University, Princeton, NJ, USA, in 2012. He was awarded Student Awards from 2001 IEEE IECON and 2003 IEEE ISIE. 